\title{
The lipid-lowering drug fenofibrate combined with si-HOTAIR can effectively inhibit the proliferation of gliomas
}

Wei Zhu ${ }^{1,2,3}$, Hongyang Zhao ${ }^{4}$, Fenfen $\mathrm{Xu}^{4}$, Bin Huang ${ }^{1,2}$, Xiaojing Dai ${ }^{5}$, Jikui Sun ${ }^{6,7,8}$, Alphonce M. K. Nyalali ${ }^{1,2,9}$, Kailiang Zhang ${ }^{1,2^{*}}$ and Shilei $\mathrm{Ni}^{1,2^{*}}$

\begin{abstract}
Background: Fenofibrate is a fibric acid derivative known to have a lipid-lowering effect. Although fenofibrateinduced peroxisome proliferator-activated receptor alpha (PPARa) transcription activation has been shown to play an important role in the malignant progression of gliomas, the underlying mechanisms are poorly understood.

Methods: In this study, we analyzed TCGA database and found that there was a significant negative correlation between the long noncoding RNA (IncRNA) HOTAIR and PPARa. Then, we explored the molecular mechanism by which IncRNA HOTAIR regulates PPARa in cell lines in vitro and in a nude mouse glioma model in vivo and explored the effect of the combined application of HOTAIR knockdown and fenofibrate treatment on glioma invasion.

Results: For the first time, it was shown that after knockdown of the expression of HOTAIR in gliomas, the expression of PPARa was significantly upregulated, and the invasion and proliferation ability of gliomas were obviously inhibited. Then, glioma cells were treated with both the PPARa agonist fenofibrate and si-HOTAIR, and the results showed that the proliferation and invasion of glioma cells were significantly inhibited.

Conclusions: Our results suggest that HOTAIR can negatively regulate the expression of PPARa and that the combination of fenofibrate and si-HOTAIR treatment can significantly inhibit the progression of gliomas. This introduces new ideas for the treatment of gliomas.
\end{abstract}

Keywords: Glioblastoma, Fenofibrate, HOTAIR, PPARA, Therapy

\section{Background}

The incidence of primary brain tumors in the world is approximately $7 / 100,000$ per year. Primary brain tumors account for approximately $2 \%$ of primary tumors that occur before the age of 70 and $7 \%$ of all cancer deaths. Glioma is the most common primary brain tumor, accounting for approximately $80 \%$ of all primary malignant

\footnotetext{
*Correspondence: Kailiang-zhang@email.sdu.edu.cn; ytfishn@126.com 'Department of Neurosurgery, Qilu Hospital, Cheeloo College of Medicine, Shandong University and Institute of Brain and Brain-Inspired Science, Shandong University, Jinan 250012, Shandong, China

Full list of author information is available at the end of the article
}

brain tumors, and glioblastoma is the most common type of glioma, accounting for more than $50 \%$ of all brain glial tumors, and is the most aggressive among all gliomas (WHO grade IV) [1]. Glioblastoma (GBM) is characterized by uncontrolled cell proliferation, diffuse infiltration, tendency for necrosis, strong angiogenesis, strong resistance to apoptosis, and genomic instability. As reflected in the old name "multiforme", GBM exhibits significant intratumoral heterogeneity at the cytopathological, transcriptional and genomic levels [1]. These characteristics make GBM one of the most difficult malignancies to analyze and treat. Despite the

(C) The Author(s). 2021 Open Access This article is licensed under a Creative Commons Attribution 4.0 International License, which permits use, sharing, adaptation, distribution and reproduction in any medium or format, as long as you give appropriate credit to the original author(s) and the source, provide a link to the Creative Commons licence, and indicate if changes were made. The images or other third party material in this article are included in the article's Creative Commons licence, unless indicated otherwise in a credit line to the material. If material is not included in the article's Creative Commons licence and your intended use is not permitted by statutory regulation or exceeds the permitted use, you will need to obtain permission directly from the copyright holder. To view a copy of this licence, visit http://creativecommons.org/licenses/by/4.0/ The Creative Commons Public Domain Dedication waiver (http://creativecommons.org/publicdomain/zero/1.0/) applies to the data made available in this article, unless otherwise stated in a credit line to the data. 
implementation of intensive treatment strategies and supportive care, the median survival of GBM has remained at 12 months during the past decade [2]. Therefore, continued in-depth study of the pathogenesis of GBM and the development of new targeted therapy methods based on its histomorphological, molecular and genomic characteristics remain crucial scientific research topics in the field of neurosurgical oncology.

In recent years, noncoding RNAs (ncRNAs) have been shown to be involved in the malignant progression of tumors and to play an important role in tumor proliferation, cell cycle progression, angiogenesis, apoptosis and invasion $[3,4]$. This type of regulatory RNA that does not encode proteins mainly includes microRNAs, lncRNAs, nucleolar small RNAs, and interfering RNAs (siRNAs). Among these RNAs, miRNAs and lncRNAs have been the most studied. miRNAs regulate gene expression at the posttranscriptional level by targeting the 3'-untranslated region (3' UTR) of specific messenger RNAs (mRNAs). IncRNAs have been shown to be related to chromosome modification, transcription and posttranscriptional regulation [5-7].

Peroxisome proliferator-activated receptors (PPARs) are members of the nuclear receptor superfamily involved in fatty acid oxidation and glucose and lipid metabolism. They are composed of three subtypes: PPAR $\alpha, \operatorname{PPAR} \beta$, and PPAR $\gamma$. PPAR $\alpha$ participates in lipid metabolism and plays an important role in regulating cell growth, differentiation, and apoptosis. Recent research results suggest that PPAR $\alpha$ is involved in the malignant progression of tumors [8]. Fenofibrate, as a PPAR $\alpha$ agonist, can inhibit the growth of gliomas in animal models of gliomas [9]. In addition, experimental results show that noncoding RNAs are involved in the regulation of the PPAR $\alpha$ signaling pathway [10]. However, the specific control mechanism remains to be further explored.

HOTAIR (Hox transcript antisense intergenic RNA) is a $\sim 2.2$-kb-long noncoding RNA transcribed from the HOXC locus [11]. Our past research confirmed that HOTAIR promotes glioblastoma cell cycle progression in an EZH2dependent manner [12]. This study was intended to explore the mechanism of lncRNA HOTAIR in regulating PPAR $\alpha$, to provide an in-depth description of the role of HOTAIR in the malignant progression of glioma by in vivo animal experiments and in vitro cell line experiments and to verify the therapeutic effect of the combination of si-HOTAIR and the PPAR $\alpha$ agonist fenofibrate on glioma cells. This study provides an experimental basis for us to understand the pathogenesis of GBM and new ideas for the molecular treatment of gliomas in clinical settings.

\section{Methods}

\section{Patient samples}

A total of 702 glioma samples from TCGA (The Cancer Genome Atlas), including 530 low-grade glioma and 172 glioblastoma samples, were used in this study. The data related to the changes in the binding of EZH2 and H3K27me3 proteins in the PPARA promoter region in glioma samples of different grades were obtained from the GEO database (GSE126396, the genome-wide distribution of EZH2 and H3K27me3 in cells at different stages of de novo neoplastic transformation was characterised by ChIP-seq, in duplicate, using an Illumina HiSeq 2500). Software IGV 2.8.6 was used for analysising database GSE126396. Glioma tissues from patients for histopathological staining were derived directly from glioma patients who underwent surgery at Qilu Hospital.)

\section{Cell culture}

The human GBM cell lines U87 and U251 were purchased from ATCC (Manassas, Virginia, USA) in August 2015. After obtaining the U87 and U251 cell lines, they were immediately cultured for proliferation and frozen in liquid nitrogen for subsequent studies. U251 cells were cultured in complete MEM (Gibco, Invitrogen, Paisley, UK) medium, while U87 cells were cultured in DMEM (Gibco, Invitrogen, Paisley, UK) medium containing $10 \% \mathrm{FBS}$ (fetal bovine serum) at $37^{\circ} \mathrm{C}$ in $5 \%$ $\mathrm{CO}_{2}$. With the exception of the in vivo cultures, all glioblastoma cells were maintained for less than eight generations.

\section{GO analysis, survival curve plot, correlation plot and heat map}

GO analysis was performed using the Cluster Profiler [13] R package. A survival curve plot was generated using the survminer (https://github.com/kassambara/ survminer/issues) R package. A correlation plot was generated using the corrplot (https://github.com/taiyun/ corrplot) $\mathrm{R}$ package. Heat map plots were built using cluster 3.0 (Stanford University).

\section{Western blot analysis}

Protein was extracted from cells that were cultured without FBS and treated with drugs following a concentration gradient for $24 \mathrm{~h}$. Equal amounts of total protein (nucleoprotein) per lane were separated using 10\% or $15 \%$ SDS-polyacrylamide gel electrophoresis and then transferred to a PVDF membrane. The membrane was blocked in 5\% skim milk for $1 \mathrm{~h}$ and then incubated with primary antibody for $2 \mathrm{~h}$. The primary antibodies used were anti-H3K27me3 (Cell Signaling Technology, USA), anti-PPAR $\alpha$ (Proteintech, Rosemont, IL) and anti-GAPD $\mathrm{H}$ (Proteintech, Rosemont, IL). Detection of protein bands was performed using a Super Signal protein detection kit (Pierce, USA). The band densities of specific proteins were quantified after normalization to the density of the GAPDH band. 


\section{Analysis of wound healing and perforation migration} U87 and U251 cells were infected with control or lentivirus containing the si-HOTAIR segment, after which the cells were treated with the presence or absence of $100 \mu \mathrm{M}$ fenofibrate (Sigma-Aldrich, Germany). Cells in each group (control, si-HOTAIR, si-HOTAIR+fenofibrate) were seeded into 6-well plates. HOTAIR siRNA segments (the HOTAIR siRNA sequence was 5'GAACGGGAGUACAGAGAGAUU-3') were obtained from GenePharma (Shanghai, China). After 24h, a $200 \mu \mathrm{l}$ sterile pipette tip was used to evenly scrape a single layer of cells. The cell debris was removed by washing with PBS (phosphate buffered saline). Then, the cells were incubated with serum-free medium under normal conditions. Photos were taken at 0 and $48 \mathrm{~h}$ after scratching. The wound area was evaluated by ImageJ, and the percentage of wound healing was calculated as follows: ( $0 \mathrm{~h}$ wound area- $48 \mathrm{~h}$ wound area) / $0 \mathrm{~h}$ wound area $\times 100$.

For the in vitro migration assays, U87-control, U87-siHOTAIR, U87-si-HOTAIR+fenofibrate, U251-control, U251-si-HOTAIR, and U251- si-HOTAIR+fenofibrate cells were seeded into the upper culture chamber of a 24-well Transwell plate. The medium in the upper chamber contained no serum, and the medium in the lower chamber contained 10\% FBS. After incubation at $37^{\circ} \mathrm{C}$ for $48 \mathrm{~h}$, nonmigrating cells on the upper surface of the membrane were removed with a cotton swab. Cells that passed through the membrane were fixed with methanol, stained with crystal violet, and counted in 5 random fields under an optical microscope (100x magnification).

\section{Colony formation test}

After treating U87 and U251 cells with control, siHOTAIR or si-HOTAIR+fenofibrate, the cells were inoculated into 6-well plates and incubated. After 14 days, the cells were fixed with $4 \%$ paraformaldehyde and stained with $0.1 \%$ crystal violet. We used a digital camera to capture pictures from different transfected colonies (more than 50 cells per clone).

\section{Chromatin immunoprecipitation (ChIP) and ChIP-qPCR assays}

ChIP assays were performed using the commercially available ChIP Assay Kit (Beyotime). The ChIP-qPCR primers used were as follows: forward-1: AACCTTGGGAGCCC CAAAAA and reverse-1: GTGCAGAGTGGTCACGTA CA; forward-2: GAGCGTGGTTTCCCAGAAGA and reverse-2: TTTGGGGCTCCCAAGGTTTT; forward-3: TACGTGACCACTCTGCACAC and reverse-3: CCTC CGGGCTCAAAGACATT; forward-4: TACGTGACCA CTCTGCACAC and reverse-4: CCTCCGGGCT CAAAGACATT.

\section{Nude mouse intracranial glioma model and in vivo bioluminescence imaging}

BALB/c-A nude mice were purchased from the Animal Center of Cancer Institute of Chinese Academy of Medical Sciences (Beijing), and their care was carried out in accordance with institutional guidelines. Three- to fiveweek-old mice were selected as the intracranial tumor model. U87 and U87-si-HOTAIR cells $\left(5 \times 10^{5}\right)$ were stereotactically injected into the right striatum of nude mice. Seven days after injection, $200 \mathrm{mg} / \mathrm{kg}$ fenofibrate suspended in 5\% sodium carboxymethylcellulose was given daily via intragastric administration in the treatment group, while an equal volume of $5 \%$ sodium carboxymethylcellulose was administered in the control group. The treatment lasted 21 days. The growth of intracranial tumors was monitored on days $7,14,21$, and 28 using a bioluminescence imaging system.

\section{Histopathological staining}

The whole brains of the mice were harvested on day 28 after the allogeneic glioma cells were implanted, fixed in 4\% paraformaldehyde, embedded in paraffin, and cut into $15 \mu \mathrm{m}$ thick coronal slices. The sections with the largest tumor area were stained with hematoxylin and eosin or were used for IHC staining.

For IHC analysis, sections were incubated with primary antibodies [1:100 dilution; anti-PPRA antibodies were purchased from Cell Signaling Technology (Proteintech), the anti-Ki67 antibody was purchased from Zsgb Bio (Beijing, China) and the anti-CD34 antibody was purchased from Abcam] overnight at $4{ }^{\circ} \mathrm{C}$, followed by a $1 \mathrm{~h}$ incubation at $37^{\circ} \mathrm{C}$ with a biotinylated secondary antibody (1:100 dilution). The samples were then incubated with horseradish peroxidase-labeled streptomycoidin and DAB (diaminobenzidine), counterstained with hematoxylin, and visualized using a light microscope.

For the IHC analysis, we quantitatively scored the tissue sections according to the percentage of positive cells and staining intensity. We assigned the following proportion scores: 1 if $0-25 \%$ of the tumor cells showed positive staining, 2 if $26-50 \%$ of cells were stained, 3 if $51-75 \%$ of the cells were stained, and 4 if $76-100 \%$ of the cells were stained; we also divided the different expression levels into four different groups (1 to 4 ) and scored them.

\section{Statistical analysis}

Statistical analyses were performed using GraphPad Prism 6.0 (GraphPad, La Jolla, CA, USA). The data are expressed as the mean \pm SD and analyzed by one-way analysis of variance for multiple comparisons or Student's t-test (two-tailed) for comparing two groups. Statistical significance was set at a value of $P<0.05$. 


\section{Results}

The expression of HOTAIR and PPARa in glioma showed a negative correlation

To verify the regulatory mechanism of the HOTAIR gene on the occurrence and progression of glioma, we heat-mapped approximately 2600 genes negatively related to HOTAIR expression. The results suggest that almost all genes were highly expressed in low-grade gliomas (Fig. 1A) and that the expression level decreased as the tumor grade increased. Further GO analysis of these genes showed that their biological processes were regulation of membrane potential, modulation of chemical synaptic transmission and regulation of transsynaptic signaling and synapse organization (Fig. 1B).

We then analyzed the signaling pathways enriched in these genes and found mainly the cAMP signaling pathway, AMPK signaling pathway and phosphatidylinositol signaling system (Fig. 1B). Most of these signaling pathways have been confirmed by scholars to be involved in the progression of tumors and different degrees of abnormal cell behaviors [14]. Excessive activation of phosphatidylinositol can lead to abnormal cell proliferation, abnormal endocytosis and exocytosis, abnormal cell metastasis and even tumor development [15]. AMP kinase promotes the bioenergy and tumor growth of glioblastomas [16].
The AMPK pathway and PPARA are both involved in metabolic activities such as fatty acid oxidation, blockade of glycolysis and protein, nucleotide and fatty acid synthesis. We further examined the expression of HOTAIR and PPAR $\alpha$ in gliomas. The results suggest that there was a significant negative correlation between them. The Pearson correlation coefficient was -0.32 (Fig. 2A). By comparing the expression levels of HOTAIR and PPAR $\alpha$ in low-grade gliomas and glioblastomas, it was found that the expression of HOTAIR in GBM was upregulated, while PPAR $\alpha$ was expressed at low levels (Fig. 2B). In the results of the survival analysis, it was found that the high expression of HOTAIR indicated a poor prognosis, but the high expression of PPARA indicated a good survival prognosis (Fig. $2 \mathrm{C}$ and $\mathrm{D}$ ). These results indicated that there is a negative correlation between the expression of lncRNA HOTAIR and PPAR $\alpha$ in gliomas and that it has significance in indicating the survival rate and clinical grade.

\section{PPARA expression decreased in clinical samples of patients with high-grade glioma}

Through survival analysis of GBM (WHO grade IV), we found that the low expression level of PPARA in the TCGA database was significantly associated with a poor prognosis for patients (Fig. 2D). To avoid bias from the

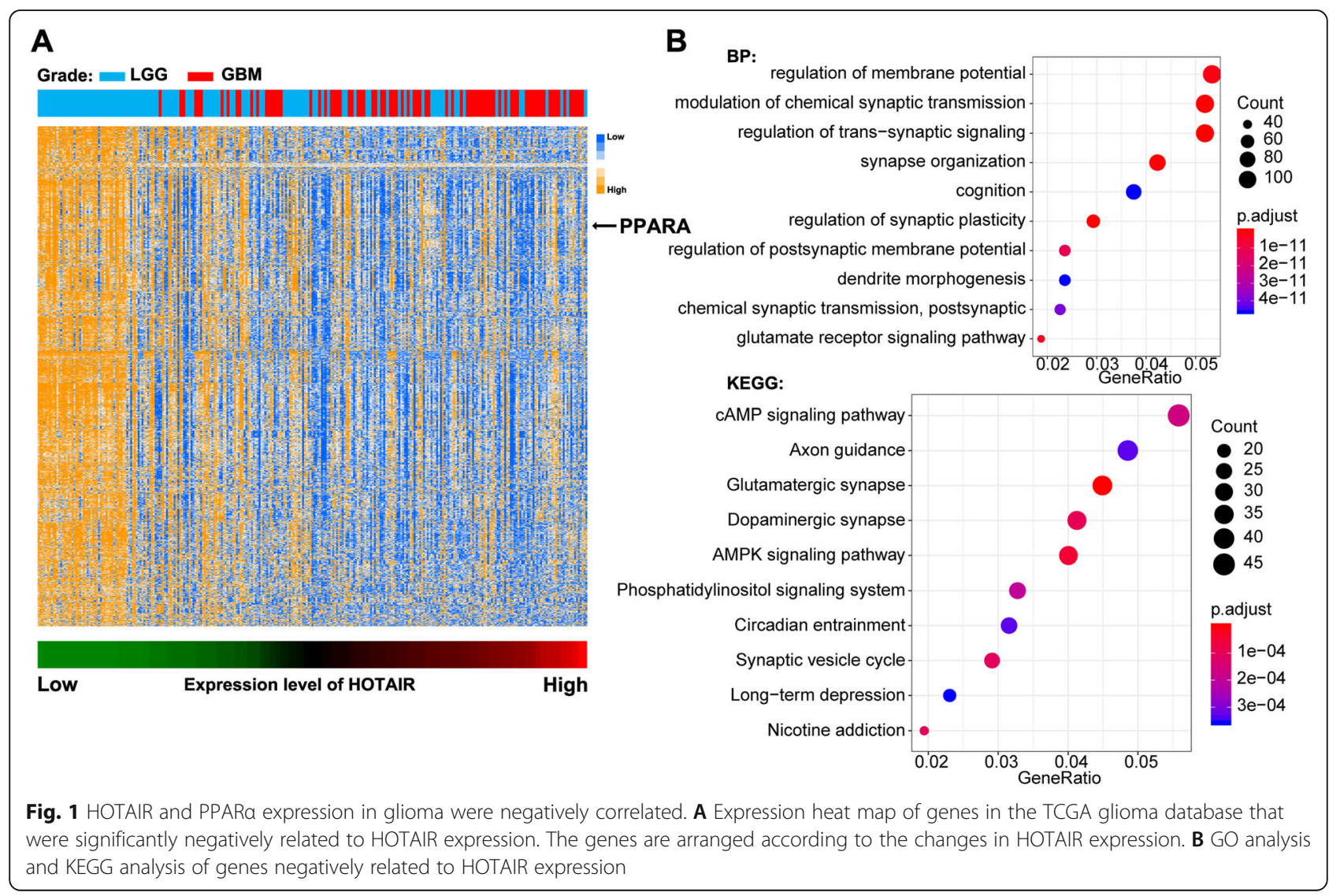




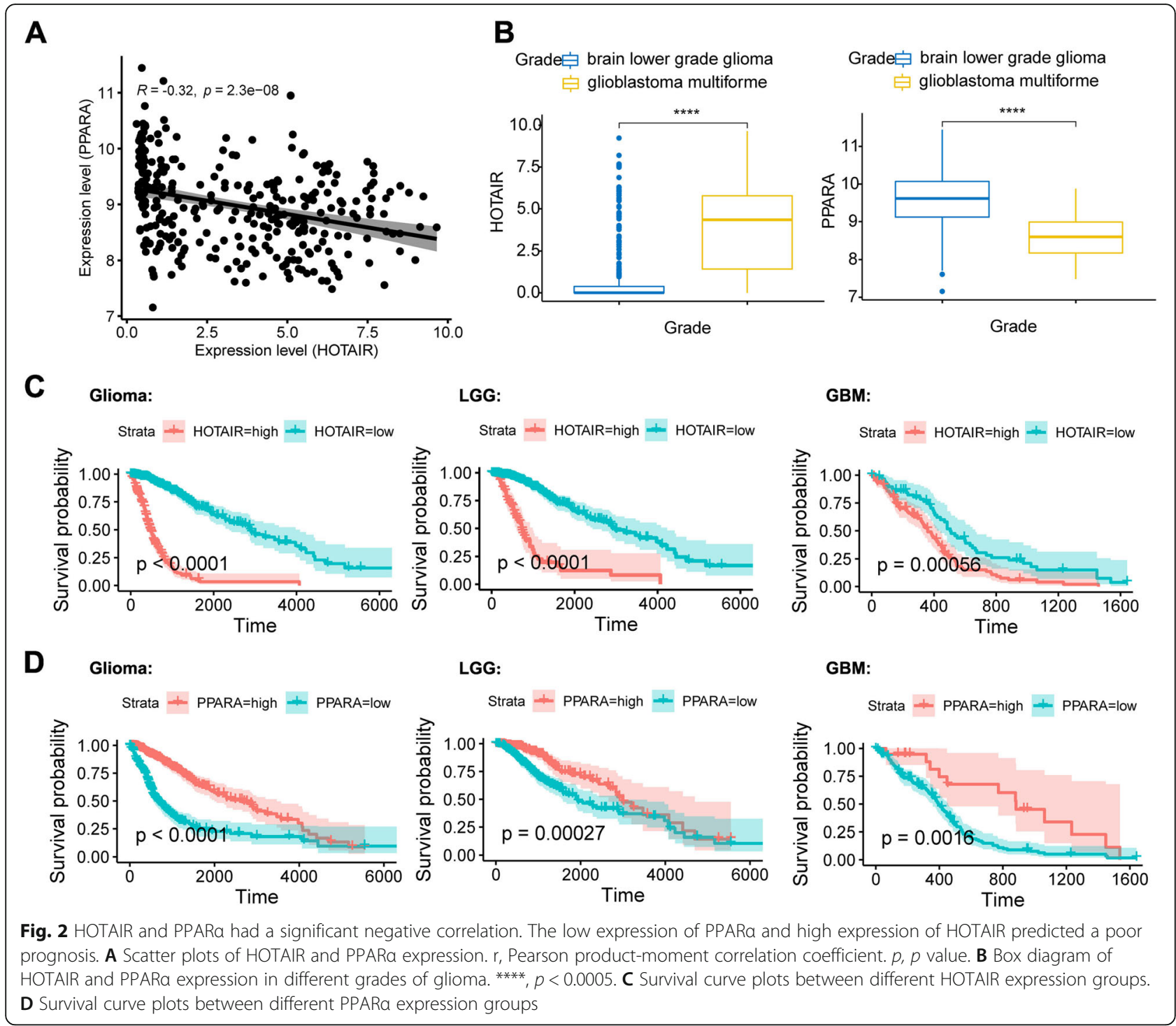

analysis of only mRNA expression data, we examined the PPARA protein levels of 21 different grades of glioma samples by immunohistochemical (IHC) staining (the clinical information is listed in Supplementary Table 1). As shown in Fig. 3A, we observed a significant decrease in PPARA protein levels in high-grade gliomas. Quantification of the staining (see Methods) revealed a strong association between the abundance of PPARApositive cells and lower tumor grade (Fig. 3B). Therefore, the downregulated expression level of PPARA is an indicator of the aggressiveness of malignant gliomas.

The expression level of HOTAIR enhanced the degree of H3K27me3 binding to the PPARA promoter

H3K27me3 is a histone modification related to transcriptional repression. HOTAIR can promote the formation of H3K27me3 through the EZH2 protein, silence the transcriptional expression of genes and promote the malignant progression of glioma. Analysis of the GSE126396 database showed that there was a significant difference in the amount of H3K27me3 protein enrichment in the PPARA promoter region of glioma samples of different malignancies (Fig. 4A). To investigate whether HOTAIR overexpression mediated PPARA promoter gene silencing, we analyzed the binding of HEK27me3 in the PPARA promoter region in control, HOTAIR and si-HOTAIR GBM cell lines by ChIP-PCR. The experimental results showed that the high expression of HOTAIR promoted the enrichment of $\mathrm{H} 3 \mathrm{~K} 27 \mathrm{me} 3$ protein in the PPARA promoter region, while reducing the expression of HOTAIR in glioma cell lines reduced the amount of binding (Fig. 4B and C). RT-PCR, western blot and immunofluorescence experiments were used to analyze the expression of PPARA 


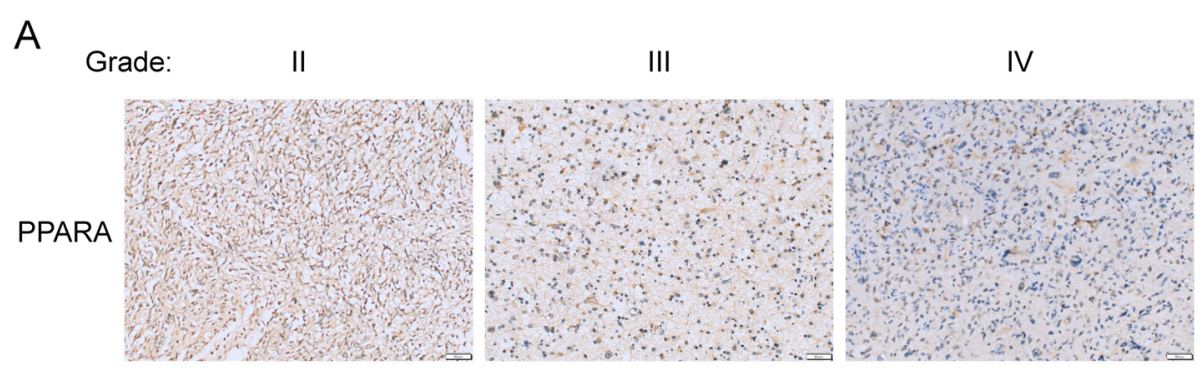

B

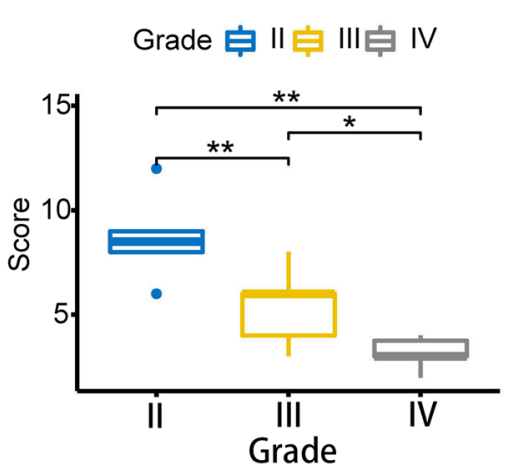

Fig. 3 PPARa expression decreased in clinical samples of patients with high-grade glioma. A PPARa immunohistochemical staining of glioma samples of different grades. B Statistical results of the immunohistochemical staining. The data are shown as the mean \pm SD. ${ }^{*}, P<0.05$; **, $P<0.01$

mRNA and protein in control, HOTAIR and siHOTAIR GBM cell lines. The expression of HOTAIR had a negative regulatory relationship with PPARA (Fig. 4D F) (Full-length blot showed in Fig. S1). Based on the above findings, we concluded that the EZH2-H3K27me3 pathway mediated by HOTAIR can silence the transcriptional expression of PPARA.

Low expression of HOTAIR and activation of the PPARA pathway weakened the migration and colony-forming ability of U87 and U251 glioma cells

To study the role of HOTAIR in gliomas, we transfected U87 and U251 glioma cells with si-HOTAIR lentivirus. Transwell migration, wound healing and colony formation analyses were performed to examine the effect of HOTAIR on glioma cell migration and colony formation. As shown by our results, U87 and U251 cells with low expression of HOTAIR showed lower migration ability than the control cells (Fig. 5A, C and E). The statistical results also showed the same results (Fig. $5 \mathrm{~B}$ and D). In further experiments, we treated si-HOTAIR group glioma cell lines with fenofibrate $(100 \mu \mathrm{M})$ and found that the cell migration and colony-formation ability were further decreased. These results indicated that the activation of the PPARA pathway can suppress the malignant phenotype of gliomas and that the combination of downregulation of HOTAIR and activation of the
PPARA pathway can more effectively inhibit the malignant progression of gliomas.

\section{Reducing HOTAIR expression in glioma cells combined with fenofibrate treatment effectively inhibited the growth of glioma xenografts}

To investigate whether reducing HOTAIR expression combined with fenofibrate treatment will reduce the growth of glioma in vivo, nude mice were implanted intracranially with U87 cells infected with a lentivirus containing a si-HOTAIR segment or a control lentivirus to form glioma xenografts. The si-HOTAIR group was randomly divided into control treatment and fenofibrate treatment groups. Seven days after injection, $200 \mathrm{mg} / \mathrm{kg}$ fenofibrate suspended in 5\% sodium carboxymethylcellulose was given daily via intragastric administration in the treatment group, while an equal volume of $5 \%$ sodium carboxymethylcellulose was administered in the control group. The treatment lasted 21 days. On days 7, 14, 21, and 28 after implantation, bioluminescence imaging of the NC, si-HOTAIR and combination therapy groups showed that the BLI signal intensity of the combination therapy group was significantly lower than that of the $\mathrm{NC}$ and si-HOTAIR groups (Fig. 6A and B). For explaining the necessity of combination medication, a separate trial was conducted between the control group using fenofibrate alone and combination group in vivo (Fig. S2). Our results suggest that combination therapy 
A

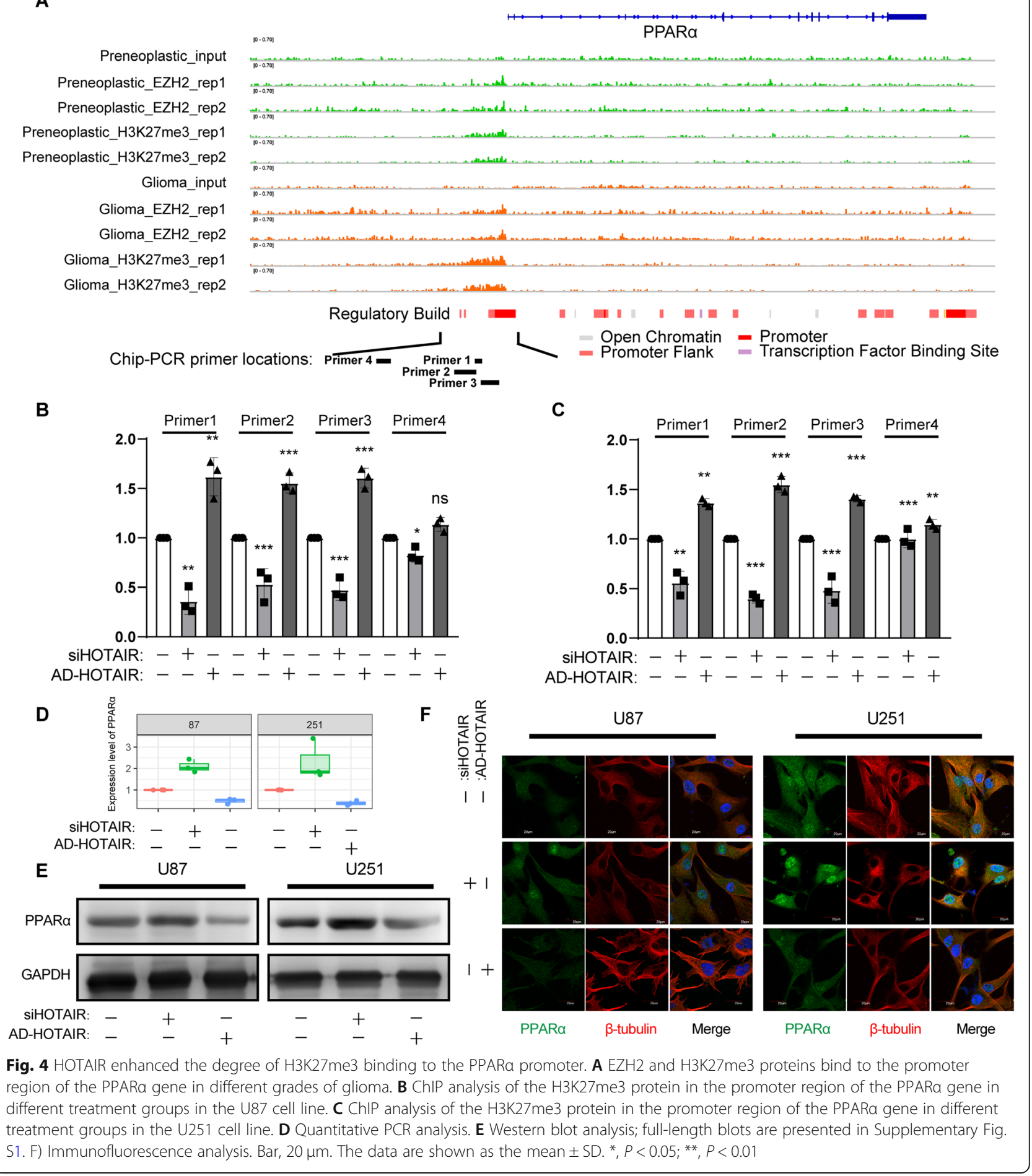

provides better treatment results than fenofibrate alone. The HE staining results were consistent with the bioimaging results (Fig. 6A). Survival analysis showed that the prognosis of the mice in the combined treatment group was significantly better (Fig. 6C). Further immunohistochemical staining showed that after reducing the
HOTAIR expression of gliomas, the expression of PPARA protein was increased, and the CD34 and Ki67 protein expression of the combined treatment group was lower than that of the other two groups. These results indicated that in an in vivo environment, reducing the expression of HOTAIR combined with treatment with 


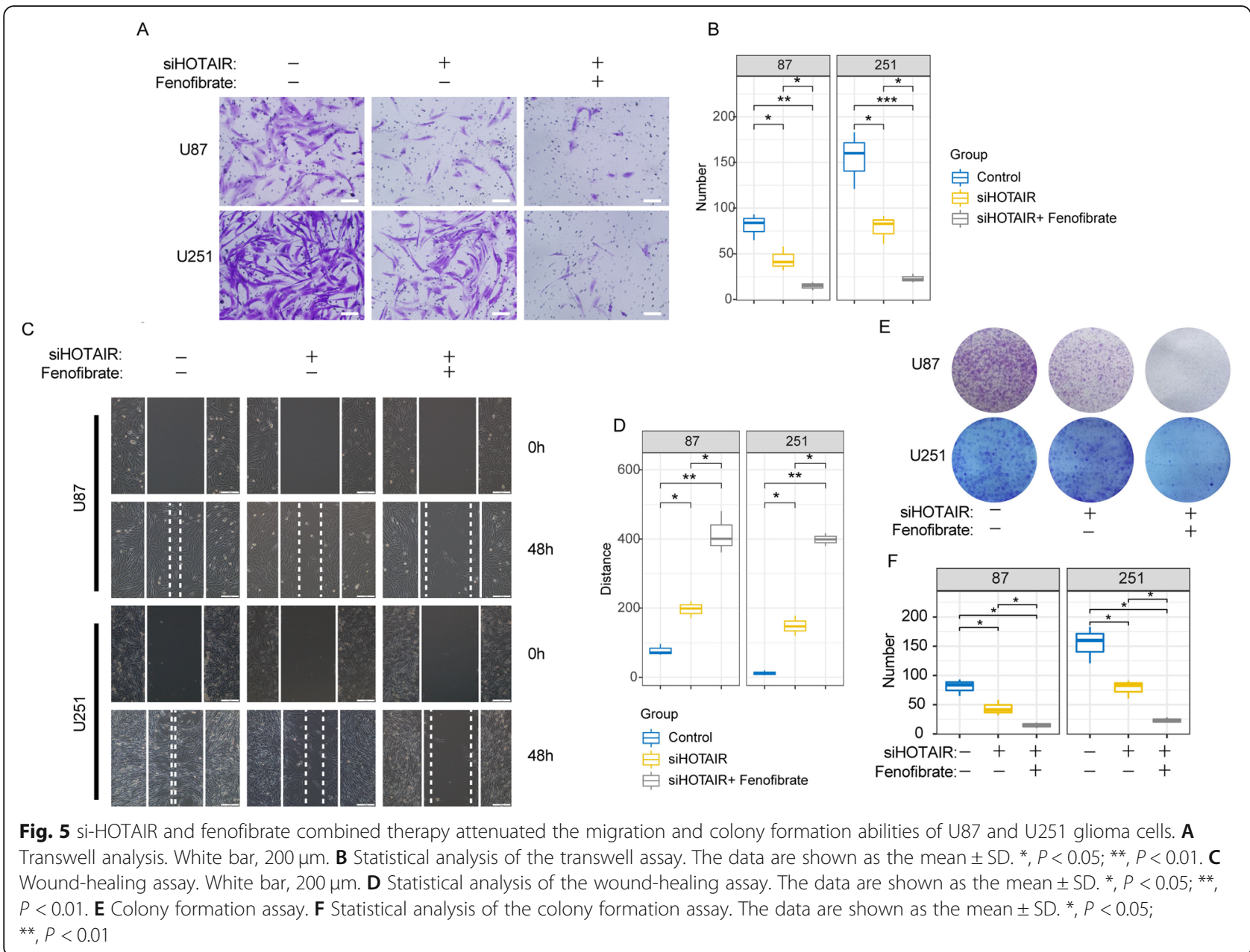

fenofibrate can effectively inhibit tumor proliferation and growth.

\section{Discussion}

GBM is the most common and deadly cancer of the central nervous system $[17,18]$. However, current conventional treatments, such as surgical resection, radiotherapy and chemotherapy, do not significantly improve the patient's prognosis. Therefore, there is an urgent need to further study the molecular pathways of glioma development and explore feasible therapeutic options. Fenofibrate is an effective ligand for peroxisome proliferator-activated receptor alpha (PPAR $\alpha)$ and has historically been used to regulate glucose and lipid metabolism in the treatment of different forms of hyperlipidemia and hypercholesterolemia [19]. In recent years, fenofibrate has proven to have interesting anticancer effects [20-24].

HOTAIR regulates the expression of a variety of proteins through PRC2 $[6,25]$. Studies by other scholars have confirmed that HOTAIR expression levels in tumors are higher than those in corresponding noncancerous tissues. Its high expression predicts a poor prognosis $[6,25,26]$. In our previous studies, HOTAIR was considered not only an important marker of tumor classification and prognosis but also an important marker of glioma molecular subtypes $[5,27,28]$.

In this study, we analyzed the expression of HOTAIR based on the RNA expression database of 702 TGGA glioma patients, analyzed the genes negatively related to HOTAIR expression using bioinformatics analyses and found that many of these genes were involved in tumor formation (Fig. 1A and B). Correlation analysis revealed that there was a significant negative correlation between HOTAIR and PPAR $\alpha$ (Fig. 2A), and low expression of PPAR $\alpha$ was shown through survival analysis to predict a poor prognosis (Fig. 2D). Through immunohistochemical staining of clinical samples, it was shown that with increasing glioma grade, the expression level of PPAR $\alpha$ decreased significantly (Fig. 3). Therefore, we predicted 


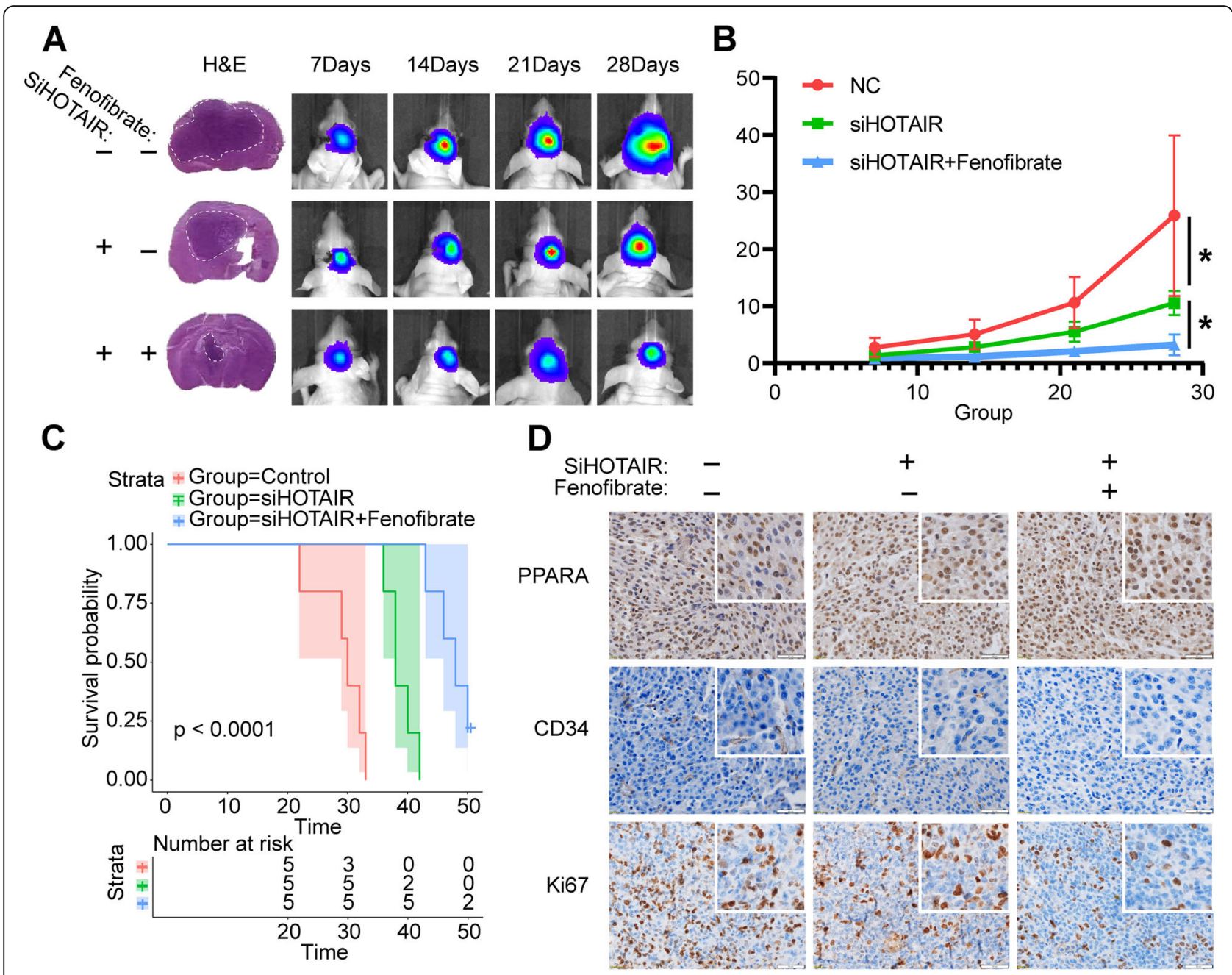

Fig. 6 si-HOTAIR and fenofibrate combined therapy slowed glioma growth. A H\&E staining of different groups of U87 intracranial glioma models. Tumor volume was measured every 7 days by in vivo imaging. B Statistical analysis results of the control and combined therapy intracranial glioma groups for every 7 days. The data are shown as the mean \pm SD. ${ }^{*}, P<0.05$. C Survival curve plots of the control and combined therapy intracranial glioma groups. D Immunohistochemical staining. White bar, $50 \mu \mathrm{m}$

that HOTAIR can reduce the expression of PPAR $\alpha$ through the PRC2 protein, thereby promoting the malignant progression of glioma.

Through ChIP-PCR analysis, it was shown that after knockdown of the expression of HOTAIR in glioblastoma, the binding of histone H3K27me3 in the promoter region of the PPAR $\alpha$ gene was significantly reduced (Fig. 4A). Through quantitative PCR, western blot and immunofluorescence, it was found that the expression of PPAR $\alpha$ increased significantly with the decrease in HOTAIR expression (Fig. 4B F). These results indicated that HOTAIR can increase the amount of H3K27me3 binding in the PPAR $\alpha$ promoter region through PRC2, thereby silencing the transcriptional expression of PPAR $\alpha$. In further in vitro migration experiments, colony formation experiments and scratch experiments, it was found that after the combination of
si-HOTAIR and fenofibrate treatment, the migration and colony-formation ability of glioma cells decreased significantly (Fig. 5). The in vivo malignant glioma model also showed that combined therapy can significantly reduce the rate of tumor cell proliferation, thereby extending the survival period of nude mice (Fig. 6).

\section{Conclusions}

Our study not only explores the molecular mechanism by which HOTAIR regulates PPAR $\alpha$ but also verifies the therapeutic effect of the combined application of the PPAR $\alpha$ agonist fenofibrate and siRNA HOTAIR on glioma through in vivo animal experiments. This provides new ideas for the comprehensive treatment of gliomas.

\section{Abbreviations}

HOTAIR: HOX transcript antisense RNA; PPARa: Peroxisome proliferator-

activated receptor alpha; IncRNA: Long noncoding RNA; GBM: Glioblastoma; 
3' UTR: 3'-untranslated region; TCGA: The Cancer Genome Atlas; ChIP: Chromatin immunoprecipitation; IHC: Immunohistochemical; PCR: Polymerase chain reaction; PRC2: Polycomb repressive complex 2; EZH2: Enhancer of zeste homolog 2; AMPK: Adenosine monophosphateactivated protein kinase; PVDF: Polyvinylidene fluoride

\section{Supplementary Information}

The online version contains supplementary material available at https://doi. org/10.1186/s12885-021-08417-z.

Additional file 1: Table S1. Information of 21 glioma samples with different grades. Figure S1. Full-length blots for blot figures in Fig. 4E. Figure S2. Fenofibrate and si-HOTAIR combined therapy slowed glioma growth. (A) Tumor volume was measured every seven days by in vivo imaging. (B) Survival curve plots of the control and combined therapy intracranial glioma groups. (C) Tumor growth curves were evaluated. The data are shown as the mean $\pm \mathrm{SD} .{ }^{*}, P<0.05$.

\section{Acknowledgments}

None.

\section{Authors' contributions}

Wei Zhu, Data curation, Writing- Original draft preparation. Hongyang Zhao, Fenfen Xu, Software. Bin Huang, Visualization, Investigation. Xiaojing Dai, Jikui Sun, Software, Validation. Alphonce M. K Nyalali, Writing- Reviewing and Editing. Kailiang Zhang, Shilei Ni, Conceptualization, Methodology, Supervision. The authors read and approved the final manuscript.

\section{Funding}

This study was supported by National Natural Science Foundation of China (Grant No. 81874082, 81702470 and 81472353), Key Research and Development Plan of Shandong Province (Grant No. 2016 GSF201014 and 2018GSF118094), Shandong Provincial Natural Science Foundation (Grant No. ZR2015HM074) and Jinan Science and Technology Development Plan (Grant No. 201821049).

\section{Availability of data and materials}

The datasets used and analyzed during the current study are available from the corresponding author upon reasonable request.

\section{Declarations}

\section{Ethics approval and consent to participate}

This study was approved by the Academic Committee of Qilu Hospital of Shandong University and was conducted in accordance with the principles expressed in the Helsinki Declaration. All datasets were obtained from the published literature, for which written informed consent was obtained.

\section{Consent for publication}

Not applicable.

\section{Competing interests}

The authors declare no competing interests.

\section{Author details}

'Department of Neurosurgery, Qilu Hospital, Cheeloo College of Medicine, Shandong University and Institute of Brain and Brain-Inspired Science, Shandong University, Jinan 250012, Shandong, China. ${ }^{2}$ Key Laboratory of Brain Function Remodeling, Qilu Hospital of Shandong University, Jinan 250012, Shandong, China. ${ }^{3}$ Department of Neurosurgery, Yantai Yuhuangding Hospital, Cheeloo College of Medicine, Shandong University, Yantai 264000, Shandong, China. ${ }^{4}$ Department of Pediatrics, Jinan Central Hospital, Cheeloo College of Medicine, Shandong University, Jinan 250013, Shandong, China. ${ }^{5}$ The Advanced Technology Genomics Core, The University of Texas MD Anderson Cancer Center, Houston, TX 77030, USA. ${ }^{6}$ School of Medicine, Nankai University, 94 Weijin Road, Tianjin 300071, China. ${ }^{7}$ Tianjin Cerebral Vascular and Neural Degenerative Disease Key Laboratory, Tianjin Neurosurgical Institute, Tianjin 30350, China. ${ }^{8}$ Department of Neurosurgery, Tianjin Huanhu Hospital, Tianjin 300350, China. ${ }^{9}$ Department of Orthopedics and Neurosurgery, Mbeya Zonal Referral Hospital and Mbeya University College of Medicine, University of Dar es Salaam, Box 419, Mbeya, Tanzania.

Received: 19 November 2020 Accepted: 24 May 2021

Published online: 03 June 2021

\section{References}

1. Rice T, Lachance DH, Molinaro AM, Eckel-Passow JE, Walsh KM, BarnholtzSloan J, et al. Understanding inherited genetic risk of adult glioma - a review. Neurooncol Pract. 2016;3(1):10-6. https://doi.org/10.1093/nop/npv02 6.

2. Furnari FB, Fenton T, Bachoo RM, Mukasa A, Stommel JM, Stegh A, et al. Malignant astrocytic glioma: genetics, biology, and paths to treatment. Genes Dev. 2007;21(21):2683-710. https://doi.org/10.1101/gad.1596707.

3. Anastasiadou E, Jacob LS, Slack FJ. Non-coding RNA networks in cancer. Nat Rev Cancer. 2018;18(1):5-18. https://doi.org/10.1038/nrc.2017.99.

4. Yang C, Wang L, Sun J, Zhou JH, Tan YL, Wang YF, et al. Identification of long noncoding RNA HERC2P2 as a tumor suppressor in glioma. Carcinogenesis. 2019:40(8):956-64. https://doi.org/10.1093/carcin/bgz043.

5. Huang K, Sun J, Yang C, Wang Y, Zhou B, Kang C, et al. HOTAIR upregulates an 18-gene cell cycle-related mRNA network in glioma. Int J Oncol. 2017; 50(4):1271-8. https://doi.org/10.3892/ijo.2017.3901.

6. Gupta RA, Shah N, Wang KC, Kim J, Horlings HM, Wong DJ, et al. Long noncoding RNA HOTAIR reprograms chromatin state to promote cancer metastasis. Nature. 2010;464(7291):1071-6. https://doi.org/10.1038/na ture08975.

7. Zhang JX, Han L, Bao ZS, Wang YY, Chen LY, Yan W, et al. HOTAIR, a cell cycle-associated long noncoding RNA and a strong predictor of survival, is preferentially expressed in classical and mesenchymal glioma. Neurooncology. 2013;15(12):1595-603. https://doi.org/10.1093/neuonc/not131.

8. Simula MP, Cannizzaro R, Canzonieri V, Pavan A, Maiero S, Toffoli G, et al. PPAR signaling pathway and cancer-related proteins are involved in celiac disease-associated tissue damage. Mol Med (Cambridge, Mass). 2010;16(56):199-209.

9. Piwowarczyk K, Wybieralska E, Baran J, Borowczyk J, Rybak P, Kosinska M, et al. Fenofibrate enhances barrier function of endothelial continuum within the metastatic niche of prostate cancer cells. Expert Opin Ther Targets. 2015;19(2):163-76. https://doi.org/10.1517/14728222.2014.981153.

10. Portius D, Sobolewski C, Foti M. MicroRNAs-dependent regulation of PPARs in metabolic diseases and cancers. PPAR Res. 2017;2017:7058424.

11. Rinn JL, Kertesz M, Wang JK, Squazzo SL, Xu X, Brugmann SA, et al. Functional demarcation of active and silent chromatin domains in human HOX loci by noncoding RNAs. Cell. 2007;129(7):1311-23. https://doi.org/10.1 016/j.cell.2007.05.022.

12. Zhang K, Sun $X$, Zhou $X$, Han L, Chen L, Shi Z, et al. Long non-coding RNA HOTAIR promotes glioblastoma cell cycle progression in an EZH2 dependent manner. Oncotarget. 2014;6(1):537-46.

13. Yu G, Wang LG, Han Y, He QY. clusterProfiler: an R package for comparing biological themes among gene clusters. Omics. 2012;16(5):284-7. https:// doi.org/10.1089/omi.2011.0118.

14. Jiang K, Yao G, Hu L, Yan Y, Liu J, Shi J, et al. MOB2 suppresses GBM cell migration and invasion via regulation of FAK/Akt and CAMP/PKA signaling. Cell Death Dis. 2020;11(4):230. https://doi.org/10.1038/s41419-020-2381-8.

15. Cheng CK, Fan Q-W, Weiss WA. PI3K signaling in glioma--animal models and therapeutic challenges. Brain Pathol (Zurich, Switzerland). 2009;19(1): 112-20.

16. Chhipa RR, Fan Q, Anderson J, Muraleedharan R, Huang Y, Ciraolo G, et al. AMP kinase promotes glioblastoma bioenergetics and tumour growth. Nat Cell Biol. 2018;20(7):823-35. https://doi.org/10.1038/s41556-018-0126-z.

17. Siegel RL, Miller KD, Jemal A. Cancer statistics. CA Cancer J Clin. 2019;2019:1.

18. Louis DN, Perry A, Reifenberger G, von Deimling A, Figarella-Branger D, Cavenee WK, et al. The 2016 World Health Organization classification of tumors of the central nervous system: a summary. Acta Neuropathol. 2016; 131(6):803-20. https://doi.org/10.1007/s00401-016-1545-1.

19. Rosenson RS. Fenofibrate: treatment of hyperlipidemia and beyond. Expert Rev Cardiovasc Ther. 2008;6(10):1319-30. https://doi.org/10.1586/14779072. 6.10 .1319

20. Hu D, Su C, Jiang M, Shen $Y$, Shi A, Zhao F, et al. Fenofibrate inhibited pancreatic cancer cells proliferation via activation of p53 mediated by upregulation of LncRNA MEG3. Biochem Biophys Res Commun. 2016;471(2): 290-5. https://doi.org/10.1016/j.bbrc.2016.01.169. 
21. Su C, Shi A, Cao G, Tao T, Chen R, Hu Z, et al. Fenofibrate suppressed proliferation and migration of human neuroblastoma cells via oxidative stress dependent of TXNIP upregulation. Biochem Biophys Res Commun. 2015;460(4):983-8. https://doi.org/10.1016/j.bbrc.2015.03.138.

22. Panigrahy D, Kaipainen A, Huang S, Butterfield CE, Barnes CM, Fannon M, et al. PPARalpha agonist fenofibrate suppresses tumor growth through direct and indirect angiogenesis inhibition. Proc Natl Acad Sci U S A. 2008; 105(3):985-90. https://doi.org/10.1073/pnas.0711281105.

23. Grabacka M, Placha W, Plonka PM, Pajak S, Urbanska K, Laidler P, et al. Inhibition of melanoma metastases by fenofibrate. Arch Dermatol Res. 2004; 296(2):54-8. https://doi.org/10.1007/s00403-004-0479-y.

24. Zhang KL, Han L, Chen LY, Shi ZD, Yang M, Ren Y, et al. Blockage of a miR21/EGFR regulatory feedback loop augments anti-EGFR therapy in glioblastomas. Cancer Lett. 2014;342(1):139-49. https://doi.org/10.1016/j.ca nlet.2013.08.043

25. Kim K, Jutooru I, Chadalapaka G, Johnson G, Frank J, Burghardt R, et al. HOTAIR is a negative prognostic factor and exhibits pro-oncogenic activity in pancreatic cancer. Oncogene. 2013;32(13):1616-25. https://doi.org/10.103 8/onc.2012.193.

26. Kogo R, Shimamura T, Mimori K, Kawahara K, Imoto S, Sudo T, et al. Long noncoding RNA HOTAIR regulates Polycomb-dependent chromatin modification and is associated with poor prognosis in colorectal cancers. Cancer Res. 2011;71(20):6320-6. https://doi.org/10.1158/0008-5472.CAN-11-1 021.

27. Wu L, Murat $P$, Matak-Vinkovic D, Murrell A, Balasubramanian S. Binding interactions between long noncoding RNA HOTAIR and PRC2 proteins. Biochemistry. 2013;52(52):9519-27. https://doi.org/10.1021/bi401085h.

28. Zhang K, Sun X, Zhou X, Han L, Chen L, Shi Z, et al. Long non-coding RNA HOTAIR promotes glioblastoma cell cycle progression in an EZH2 dependent manner. Oncotarget. 2015;6(1):537-46. https://doi.org/10.18632/ oncotarget.2681.

\section{Publisher's Note}

Springer Nature remains neutral with regard to jurisdictional claims in published maps and institutional affiliations.

Ready to submit your research? Choose BMC and benefit from:

- fast, convenient online submission

- thorough peer review by experienced researchers in your field

- rapid publication on acceptance

- support for research data, including large and complex data types

- gold Open Access which fosters wider collaboration and increased citations

- maximum visibility for your research: over $100 \mathrm{M}$ website views per year

At $\mathrm{BMC}$, research is always in progress.

Learn more biomedcentral.com/submissions 